\title{
SOBRE UNA ALTERNATIVA NOMINALISTA A LA SEMÁNTICA DE FREGE-CHURCH
}

\section{Introducción}

En The Diversity of Meaning, ${ }^{1}$ el profesor L. J. Cohen presenta un' análisis nominalista de los contextos no extensionales, análisis al que denomina "teoría de los operadores"; esta última es expuesta en contraste sistemático con la semántica de Frege-Church, a fin de poner de relieve sus ventajas desde el punto de vista del poder explicativo y de su compatibilidad con las intuiciones del sentido común. El objeto de este artículo es mostrar que la teoría de Cohen adolece de serias deficiencias y que algunos de sus análisis son insostenibles; en algunos puntos se hará una detallada comparación de ambas teorias (cf. $\$ \S 5,6$ ), lo que dará lugar a consideraciones de alcance más general acerca de los requisitos mínimos que debe satisfacer un análisis nominalista y la naturaleza de la semántica platonista de Frege.

Según el profesor Cohen, lo que se necesita para la solución de los problemas planteados por lá no extensionalidad es "una teoría general de los operadores que forman enunciados [statements] a partir de 'decires' [sayings]", donde un enunciado es un 'decir' verdadero o falso (p. 201). Por el momento bastará señalar que un 'decir' no es otra cosa que 'un token oracional [sentence-token] descrito bajo un determinable" (p. 166), el cual, según se supone, representa "lo que un hombre puede repetirse a sí mismo, comunicar a otros, o considerar ya como premisa, ya como conclusión” (p. 163). No hay en la comunicación verbal intercambio alguno de significados concebidos como entidades platónicas que se ocultan bajo el disfraz lingüístico:

Cuando Fulano se repite a sí mismo el consejo de un amigo, o transmite un informe de éste, el sentido en el cual lo que Fulano dice es lo mismo que oyó antes es el sentido en el cual el token oracional emitido por Fulano tiene al menos una propiedad - la de ser un 'decir' tal y tal [the property of being such and such a saying]_- que también posee el token oracional producido por su amigo (p. 166).

1 London, Methuen and Co. Ltd., $2^{\text {a }}$ ed., 1966, pp. xii, 369 .

2 Aclaraciones terminológicas: a) la palabra "decir" irá con comillas simples cuando sea usada como sustantivo, a fin de evitar que ciertos párrafos resulten demasiado confusos al lector no advertido; b) por razones de brevedad empleo la palabra inglesa "token" en lugar de su posible traducción como "expresión-suceso", que complicaría aún más algunas oraciones cuyo inevitable retorcimiento no deseo agravar. Aunque no propongo generalizar el procedimiento, es obvio que la traducción de "token" como "token" posee la ventaja de una extraordinaria fidelidad al texto inglés. 
EI autor considera los 'decires aseverativos' [assertive sayings = statements, en su terminología] como los objetos de las actitudes proposicionales, lo que concuerda con su rechazo de las entidades subsistentes en semántica; los tokens correspondientes a palabras o frases que componen un 'decir' son llamados "términos" (p. 162). Un operador que forma enunciados es simplemente una expresión que gobierna una cláusula subordinada: "es necesario que", "es una ley causal que", etc., son ejemplos de tales operadores. Finalmente, el profesor Cohen distingue entre un 'decir' y su significado, por razones que omitiremos en este momento, pero deberemos considerar más adelante.

La teoría propuesta por Cohen presenta dos niveles diferentes de análisis, a los que nos referiremos como Análisis 1 y Análisis 2. En virtud del primero

es conveniente considerar que todos los casos de no extensionalidad surgen debido a la presencia implícita o explícita de operaciones que for: man enunciados a partir de 'decires' (p. 201).

A fin de justificar este diagnóstico general acerca del origen de la no extensionalidad, el autor se ve compelido a introducir algunas equivalencias de significado que pondrían al descubierto los operadores ocultos requeridos por su teorfa. Así, supone que la oración:

(1) La característica de ser un bípedo implume no es idéntica a la característica de ser un animal racional

posee un significado equivalente [is equivalent in meaning] a:

(2) No es analítico que todos los bípedos implumes y sólo ellos sean animales racionales,

donde (2) trae a la luz del día el operador "No es analítico que", el cual vivía de incógnito en (i). Cuando este tipo de transformación no resulte posible, como en lọs casos de cita directa mediante comillas, Cohen sostendrá que no se trata de un genuino ejemplo de no extensionalidad (cf. $§ 7$ ). Según la teoría propuesta, la no extensionalidad se explica fácilmente por el hecho de que la sustitución de una expresión por otra puede cambiar la identidad del 'decir' (cf. $§ 5$ ). Pero

la reducción de todos los contextos no extensionales a enunciados que contienen operadores que forman enunciados a partir de 'decires' [Análisis 1] es sólo una primera etapa. Existe una forma de enunciado lógicamente más simple que es equivalente a la primera. Para cualquiera de esos enunciados hay otro de significado equivalente que predica algo 
acerca de o bien algunos o todos los tokens del 'decir' que lo constituye (p. 207).

Esta forma "lógicamente más simple" de enunciado es esencialmente una versión modificada del análisis de los enunciados de aseveración y creencia propuesto por Scheffler; ${ }^{3}$ según esta versión, una oración como:

(3) Un cretense dice que está lloviendo

se transforma en:

(4) Hay un token $x$ tal que ( $x$ es un enunciado dicho por un cretense y $x$ es un enunciado de que está lloviendo) y ( $x$ es verdadero si y sólo si está lloviendo).

Este ejemplo muestra la naturaleza del Análisis 2. Omitimos la transformación ulterior que debiera sufrir la oración (2), si la sometemos a este segundo análisis, pues no nos ocuparemos aquí de tal tipo de enunciados.

Un rasgo importante de la teoría de los operadores es que no requiere ningún cambio de la referencia y el significado normales de las expresiones que figuran en un contexto no extensional, cambio característico de la semántica de Frege y mediante el cual éste pretendió salvar la ley de extensionalidad:

Si suponemos que, explícita o implícitamente, todos los casos de no extensionalidad informal surgen cuando se agregan a los 'decires' ciertos operadores, debemos suponer que la identidad de los 'decires' no es afectada por tal agregado. El mismo 'decir' figura, ya en un contexto no extensional, ya en uno extensional, y puesto que los 'decires' son los mismos también lo son sus significados y el significado de sus términos (p. 206).

Como era de prever, el autor no hace intento alguno por salvar la ley de extensionalidad:

En lugar de preservar la ley de extensionalidad para los enunciados no formalizados mediante la modificación de su interpretación normal [como en la semántica de Frege], la teoría de los operadores mantiene, en cambio, la interpretación normal de estos enunciados y se limita a aceptar que la ley de extensionalidad no se aplica a ellos ni siquiera en su forma más débil (p. 207).

La referida versión débil de la ley de extensionalidad —o sea la que se

3 I. Scheffler, "An Inscriptional Approach to Indirect Quotation" (Analysis, vol. 14, No 4, 1954, pp. 83-9o), y Anatomy of Inquiry, New York, Alfred A. Knopf, 1967, pp. 88-110. 
salvaría por el análisis fregeano- es presumiblemente una versión contextual de la ley de Leibniz: no dice que si ' $a=b$ ' es verdadera entonces $a$ y $b$ son intercambiables salva veritate en cualquier contexto, sino que son intercambiables si tienen la misma denotación dentro de los contextos en los cuales se efecúa el remplazo. Volverèmos a esta cuestión más tarde, al examinar el tratamiento dado por el autor a los contextos de comillas (\$7).

En contraste con ello y a modo de compensación, el autor anuncia que

en el plano de la formalización la teoría de los operadores permite un grado de extensionalidad mayor que Frege. La lógica general de los operadores que forman enunciados a partir de 'decires' puede ser representada en un sistema formal en el cual la ley de extensionalidad es demostrable en su versión más fuerte (p. 207).

Como trataremos de mostrar, sin embargo, la extensionalidad fuerte admitida por el sistema formal de Cohen es el simple resultado de una restricción ad hoc sobre los casos' de sustitución de las variables predicativas' y carece de todo valor clarificatorio para el análisis de los enunciados del lenguaje ordinario (cf. $\$ 6$ ).

\section{Identidad de sentido e identidad de 'decires'}

Al criticar a Frege, Cohen afirma con razón que Frege no proporciona ningún criterio para la identidad de sentido, dejando esta noción básica en la oscuridad, 4 y sostiene que cualquier criterio concebible que pudiera proponerse resulta ser o bien más fuerte o bien más débil de lo qué se necesita en relación con algunos tipos de inferencia. No me detendré aquí en los ejemplos dados por Cohen. (pp. 195-7), ni sugeriré que la teoría de Frege puede lidiar con ellos. Sólo me interesa mostrar que la posición de Cohen es en este respecto tan infortunada como la de Frege, lo cual surge claramente de su propia exposición.

Sería incorrecto afirmar, sin embargo, que Cohen no ofrece criterio alguno para la identidad de 'decires'; el criterio que ofrece es el siguiente:

(C) Dos tokens son el mismo 'decir' [...] si y sólo si tienen el mismo significado $[\ldots]$ y o bien pueden ser reformulados con las mismas palabras [admit of identically worded reformulations] o uno es él mismo una reformulación del otro en discurso indirecto (p. 162).

4 Cf., sin embargo, Christian Thiel, Sense and Reference in Frege's Logic (D. Reidel Publishing Company, Dordrecht, Holland, 1968), p. 131. En una carta a Husserl citada por Thiel, Frege formula explicitamente un criterio de identidad, basado en la noción de equivalencia lógica; pero este criterio no es satisfecho siquiera por sus propios ejemplos de expresiones no sinónimas. 
Dejando a un lado por ahora el requerimiento adicional de identidad de significado, la clave de la cuestión es ésta: ¿en qué condiciones puede afirmarse, dados dos tokens, que uno es una reformulación del otro en discurso indirecto? Si conociéramos la respuesta no tendríamos ya problema alguno, como lo muestra la patética historia del isomorfismo intencional de Carnap y otros intentos similares.

Sería demasiado engorroso y poco gratificante detenerse en los ejemplos dados por el autor, pues la dificultad que afecta la teoría de los operadores se revela de modo concluyente en sus explicaciones generales. El autor explica que

en la individuación de los 'decires' las palabras que los componen [their wording] son tan importantes como su significado, pero lo son de un modo tal que no sólo no cuentan las diferencias de lenguaje y las variaciones triviales de las frases [...] sino tampoco ciertas diferencias en el contexto inmediato de su emisión (p.164).

En conexión con un ejemplo de dos oraciones diferentes que a juicio del autor representarían un mismo 'decir', Cohen afirma que tales oraciones "sólo difieren en ciertos respectos permitidos" (p. 164; el subrayado es mío), pero nada agrega en este punto para clarificar el carácter de tal 'permisividad lógica'; es fácil suponer, sin embargo, que una diferencia en las palabras componentes es 'permitida' si preserva la condición de ser una 'reformulación', de modo que estaríamos nuevamente en el punto de partida. (Es interesante observar que la misma cuestión podría plantearse en relación con el concepto de 'rephrasal' propuesto por Scheffler, ${ }^{5}$ con la reserva, sin embargo, de que Scheffler no pretende poseer la clave de la solución; lo que él sostiene es que, considerando la conocida debilidad del análisis platónico en cuanto al problema de hallar un criterio de identidad para las proposiciones [en el sentido de Frege] el nominalista tiene el derecho de proponer un análisis alternativo sin verse obligado por ello a ofrecer un criterio que nos permita establecer cuándo una inscripción es 'a rephrasal' de otra.) ${ }^{6}$

Para colmo de males, el mismo autor se encarga de señalar que el mencionado criterio de identidad de 'decires' puede dar pie al cargo de circularidad:

Es cierto que la identidad de reformulación en discurso indirecto fue propuesta $[\ldots]$ como un criterio para la identidad de 'decires', y esto

5 Cf. la primera referencia en nota 3 .

6 Para decirlo con palabras de Quine: "Let it not be objected just here that there is no evident way of telling how far an utterance may be allowed to differ from the sentence appearing in the position of ' $p$ ' and still be counted as an utterance that $p$. The objection is correct $(\ldots)$ and Scheffler appreciates it. But it is out of order just now, for it concerns indirect quotation however analysed, and has no special. relevance to Scheffler's contribution" (World and Object, p. 215). 
podría sugerir que cualquier análisis del discurso indirecto en términos de enunciados acerca de enunciados debe ser circular (p. 208).

Recordemos, en efecto, que la falla de la sustituibilidad en el discurso indirecto fue explicada por el hecho de que la sustitución cambia la identidad del 'decir':

Cámbiense las palabras de una oración en cualquier aspecto que no sea trivial [in any other than trivial respects] y ya no podrá ser descrita verídicamente como un 'decir' emitido por cierta persona en cierta ocasión (p. 211 ).

De acuerdo con el autor, sin embargo, la teoría de los operadores puede eliminar fácilmente tal circularidad. El examen del medio propuesto por Cohen para saltar fuera del círculo posee un interés adicional, y es que si realmente pudiéramos saltar, dispondríamos también de un criterio efectivo para la identidad de 'decires', como se desprende del texto siguiente:

Pero la circularidad puede evitarse fácilmente si, en vez de referirnos a la reformulación en discurso indirecto, confeccionamos una lista detallada de aquellas variaciones en las palabras que requiere normalmente la reformulación en discurso indirecto, tal como el cambio de pronombre y la forma del verbo en ciertos casos, y estipulamos que sólo estas variaciones dejan inalterada la identidad del 'decir' (p. 208; el subrayado es mío).

No cabe duda alguna de que Cohen puede estipular que los cambios usuales en los pronombres y en las formas verbales son los únicos 'permitidos', pero difícilmente sería considerado esto como una clarificación del problema; pues aun en el caso de que tal lista reflejara fielmente las variaciones de verbos y pronombres efectuadas en el lenguaje ordinario, quedaría el problema de las posibles variaciones de predicados y nombres. Puede ser que, como Cohen afirma, "todo lo que se requiere son listas de instrucciones para el ejercicio escolar de poner oratio recta en oratio obliqua"; pues si realmente tuviéramos la lista requerida, podríamos dársela a los niños como entretenimiento, lo que quizás redimiría a la semántica de su deshonrosa esterilidad. Pero es engañoso sugerir que es fácil confeccionar tal lista; ¿incluiría Cohen en ella, por ejemplo, la transformación de la voz activa en la pasiva? El cambio requerido en este caso es tan simple que puede ser efectuado por cualquier persona que posea un conocimiento básico del lenguaje, pero su interpretación semántica es un asunto diferente, como lo prueba una larga y aún no concluida discusión. ${ }^{7}$ Debido a estas dificultades, no es demasiado reconfortante enterarnos de que

7 Cf. Paul Ziff, "The Nonsynonymy of Active and Passive Sentences" (Philosophical 
si llega a descubrirse un nuevo lenguaje, la definición de "ser el mismo 'decir'" puede ampliarse de manera apropiada (p. 208).

\section{Significado y palabras indicadoras}

La relación entre un 'decir' y su significado adquiere un carácter extremadamente dudoso cuando entran en escena las palabras indicadoras (indicattor-words). Según la p. 162, las afirmaciones

(i) Usted parece cansado

(ii) Earece cansado

poseen el mismo significado si los tokens respectivos de "usted" y "él" hacen referencia a la misma persona en un determinado contexto de emisión; y dos tokens diferentes de

(iii) Yo tengo ojos cafés,

producidos respectivamente, digamos, por usted y por mí, poseen un significado diferente. Esto implica que los tokens correspondientes de "usted" y "él" tienen el mismo significado en tal ocasión, y que los dos tokens de "Yo", en cambio, poseen un significado distinto. El autor dice explíctamente, en relación con este último caso, que el token de "Yo" producido por usted es un término diferente del producido por mí, "debido a que sus significados difieren" (p. 143).

En apoyo de su opinión, Cohen observa que

your remark means that you have brown eyes, and mine means that I have (loc. cit.; el subrayado es mío).

En cierto sentido es indudablemente así; pero es bien conocido que el verbo inglés "to mean" no es lógicamente confiable, en virtud de sus diversos usos, y que el no meditado salto de "to mean" a "meaning" puede ser mortal. Si alguien afirma, para dar un ejemplo extremo: "When I spoke of 'that fat charlatan' I meant Smith", 8 la veracidad de su afirmación no implica que Smith en persona fue el significado (meaning) de la expresión empleada. De modo que este verbo sospechoso no basta para justificar la diferencia de significado entre los dos tokens de "Yo", a menos que Cohen

Review, LXXV, 1966, pp. 226-232); también la réplica de J. J. Katz y E. Martin, “The Synonymy of Actives and Passives" (Phil: Review, LXXVI, 1967, pp. 476-91).

8 Debo este ejemplo a G. E. M. Anscombe, An Introduction to Wittgenstein's Tractatus, London, Hutchinson and Co. Ltd., $3^{\text {a }}$ ed., 1967 , p. 17. 
esté dispuesto a identificar el significado con la referencia, lo que de hecho no ocurre. ${ }^{9}$

En la terminología usual se diría que las dos mencionadas emisiones de (iii) producen dos enunciados (statements) diferentes con el mismo significado; y ello implica que el conocimiento del significado no requiere información fáctica, concerniente, por ejemplo, al contexto referencial. Si oigo dos emisiones de (iii) a través de una pared, captaré su significado lingüístico aun cuando ignore que cada una de ellas fue producida por una persona diferente; la falta de la información fáctica necesaria para saber a quién se referían no me impedirá traducirlas al francés, un hecho que concuerda curiosamente con el consejo de Cohen en otra parte de su libro: "Don't ask for the meaning; ask for translation or paraphrase" (p. 149). En este caso la situación es similar a la planteada por dos tokens de "El actual rey de Francia es calvo" cuando son producidos en épocas distintas; y Cohen no hablaría aquí de una diferencia de significado.

No deseo negar por supuesto, que si tanto $X$ como $Y$ producen un token de (iii), entonces lo que dice $X$ es, en cierto sentido, diferente de lo que dice $Y$; pero esta diferencia obvia en lo que es dicho no puede elucidarse mediante la supuesta diferencia de significado.

Cohen agrega que "las reglas para evaluar la verdad de la afirmación hecha por usted o para argumentar a partir de ella difieren de las reglas por aplicar en relación con la afirmación hecha por mí" (pp. 162-3); es posible que así sea, pero lo mismo vale para los mencionados tokens de "El actual rey de Francia es calvo".

Consideraciones similares son también aplicables a los casos (i)-(ii), o sea a la supuesta identidad entre los significados de "El" y "Usted" cuando se usan para hacer referencia a la misma persona. Al parecer, sin embargo, el criterio (C) obliga a Cohen a sostener que son idénticos, pues desea considerar que las emisiones correspondientes de (i) y (ii) producirían el mismo 'decir': “Mi observación a Smith: 'Usted parece cansado', y la efectuada por usted [...] en la misma ocasión: 'Él parece cansado', son el mismo 'decir', porque de ambos [de usted y de mí] podría afirmarse que hemos dicho que él parecía cansado" (p. 162).

9 Cohen dice, en efecto: "Not that their meanings are the people they severally refer to, for their meanings do not walk about, wear clothes and eat food" (p. 16g; el subrayado es mío). Deseo sugerir incidentalmente aquí que el argumento de tipo wittgensteniano destacado en cursiva, dirigido usualmente contra la semántica denotacionalista, es impresionante pero inconcluyente. Las razones de Frege son mejores. Una muy elaborada teoría denotacionalista del significado puede verse en J. Hintikka, "Semantics for propositional attitudes" (Models for Modalities, D. Reidel Publishing Company, Dordrecht-Holland, 1969, pp. vii, 220). 


\section{Sobre algunos enunciados indóciles}

Una situación algo curiosa surge en relación con un tipo de enunciados que Quine presenta en Word and Object (p. 215) como una "peculiar dificultad" para el análisis de Scheffler, pero que Cohen declara haber forzado a entrar en el redil nominalista, lo que sería un trofeo no despreciable para la teoría de los operadores. Consideremos la oración:

(1) Juan cree algo que Pedro no cree,

que en términos platonistas podría simbolizarse como

(2) (A $p)[B(x, p) . \sim B(y, p)]$,

donde " $B(x, p)$ " puede leerse " $x$ cree $p$ ".

Quine observa correctamente que Scheffler no puede aplicar a (1) la transformación nominalista que propone para otros casos: “... no servirá decir que Juan cree-verdadera ${ }^{10}$ alguna inscripción que Pedro no cree-verdadera, pues puede ocurrir que no exista ni llegue a existir nunca una inscripción tal; el creer, contrariamente al decir, no produce emisiones [utterances]".

Cohen propone, sin embargo (p. 228), la siguiente interpretación nominalista de (1):

(3) $($ G $F)((w)(F w \equiv S w) .((x)(F x \supset B x y) . \sim(\exists x)(F x . B x z)))$,

donde " $\mathrm{S}$ " representa "cierto particular enunciado" [a certain single state$m e n t]$ y "Bxy" es una abreviatura de " $x$ es creído por $y$ ".

Es natural sentir que la presencia de "( $(\mathrm{T} F)$ " en (3) es un comienzo escandaloso para un análisis nominalista; pero no nos detendremos en ello ahora. Puede esgrimirse contra (3) una objeción más específica: la de que podría ser verdadera aun en el caso de que el universo careciera de tokens y $F$ fuera una propiedad vacía; en tal caso " $S w$ " y " $F w$ " serian siempre falsos, y en consecuencia " $(x)(F w \equiv S w)$ ", " $(x)(F x \supset B x y)$ " y " (耳 $x)(F x$. $B x z)$ " serían siempre verdaderos. Es obvio sin embargo que la ausencia de emisiones lingüísticas no debiera ser suficiente para la verdad de (1) en un análisis que concibe la creencia como una relación entre personas y emisiones.

Pero aun si pasáramos por alto la fantasía de un universo carente de tokens, argumentando que el mero hecho de pronunciar o escribir (3) nos proporciona al menos uno, no garantizaría ello la existencia del 'particular

10 Siguiendo una sugerencia de Quine en "Quantifiers and Propositional Attitudes", (The Way of Paradox, New York, Random House, 1966), Scheffler usa el predicado "creeverdadera" para una relación entre personas e inscripciones, en vez de la expresión "cree que". 
enunciado' que se requiere, pues es claro que lo que se necesita es 'cierto enunciado' y no uno cualquiera (en caso contrario (1) significaría que $y$ cree todo y $z$ nada). De este modo nos quedamos otra vez sin el relatum nominalista de la creencia.

Podría replicarse, sin embargo, que hemos entendido mal el significado de "( (马 $F)$ " y que el significado que el autor dio explícitamente a este símbolo impide que (3) pueda ser vacuamente verdadera (en el sentido explicado); según Cohen, en efecto, "es conveniente suponer que una propiedad existe si y sólo si existe un ejemplo de ella" (p. 224). Pero esta estrategia sólo puede resultar convincente cuando tratamos con una propiedad específica, o sea cuando " $F$ " es una constante predicativa; pues en este caso es posible introducir la definición $\mathrm{g} F=\mathrm{df}$ ( $\mathrm{G} x$ ) $F x$, donde el primer " $\mathrm{g}$ " no es un cuantificador sino un predicado de segundo nivel. Pero cuando " $F$ " es una variable no es posible efectuar esta jugada deflacionista; las declaraciones no platónicas de Cohen carecen de un apoyo adecuado, y no son siquiera formuladas mediante la notación cuantificacional corriente. Ésta es, por supuesto, una observación que lleva la impronta de Quine, y sirve para justificar el sentimiento expresado antes acerca de la escandalosa presencia de "( $($ I $F)$ " en un análisis nominalista.

Por otra parte, podría sugerirse que (3) debiera transformarse en:

$$
\text { (4) (马 } F)((w)(F w \equiv S w) \text {. (马 } x)(F x . B x y) \text {. } \sim(\text { (马 } x)(F x . B x z) \text {; }
$$

pero entonces sería evidente que la dificultad planteada por Quine se aplica por completo a (4), pues su falsedad es perfectamente compatible con la verdad de (1), en vista del hecho de que el token cuya existencia se afirma no es mostrado en (4). La adecuación de un análisis nominalista requiere al menos que la correspondencia de valores veritativos entre el analisandum y el analisans quede asegurada por la existencia de los tokens requeridos; si la pretensión de que existen no puede ser fundada de algún modo, la posibilidad fáctica de una divergencia de los valores veritativos quedará abierta. Podemos concluir, entonces, que ni (3) ni (4) bastan para vencer la resistencia antinominalista de (1).

\section{Sustituibilidad y forma lógica}

Como ya sabemos, Cohen sostiene que la teoría de los operadores puede explicar fácilmente "por qué hay tantos grados de no extensionalidad en el discurso informal”. La explicación es simple:

Aplicamos a las cosas predicados diferentes en virtud de las diferentes características poseídas por ellas, así como "caliente", "frío", etc., se aplican a un objeto en virtud de su temperatura, y "rojo", "amarillo", 
etc., en virtud de su color. Cámbiese la temperatura de un objeto más allá de cierto límite, y puede no ser ya correcto describirlo como caliente; cámbiense las palabras de una oración en aspectos no triviales y puede no ser ya correcto describirla como el 'decir' producido por cierta persona en cierta ocasión (p. 211).

No negaré la plausibilidad intuitiva de este diagnóstico general, que puede reformularse fácilmente en términos fregeanos. Considerando que en la semántica fregeana un enunciado de aseveración o creencia establece una relación entre una persona y una proposición (nombrada por la cláusula que comienza con el relativo "que"), podría también decirse, parafraseando a Cohen, pero desde un punto de vista fregeano: "Aplicamos a las proposiciones predicados diferentes en virtud de las diferentes características poseídas por ellas... Cámbiense las palabras de una oración en aspectos no triviales y puede no ser ya correcto describirla como expresando la proposición afirmada (o creída, etc.), por cierta persona en cierta ocasión". Y como en el sistema intensional de Church los operadores modales son simplemente predicados aplicables a proposiciones, disponemos de una explicación similar para la falla de la sustituibilidad en los contextos modales.

En lo que sigue veremos, sin embargo, que dentro del esquema semántico de Cohen la referida plausibilidad es sólo un fuego fatuo, debido a la falta de un soporte teórico adecuado. En esta sección plantearé una dificultad vinculada con el principio de indiscernibilidad de los idénticos; otra dificultad, de un carácter quizás más fundamental, será examinada al final de $\S 6$.

Consideremos los enunciados verdaderos:

y

(1) Jorge IV dudaba de que Scott $=$ el autor de Waverley

(2) Scott $=$ el autor de Waverley.

Pese a la verdad de (1)-(2), la sustitución de "el autor de Waverley" por "Scott" produce la falsedad:

(3) Jorge IV dudaba de que Scott $=$ Scott.

Según la teoría de los operadores este cambio del valor veritativo no es sorprendente, debido a que (el token de) "Scott $=$ el autor de Waverley" no es el mismo 'decir' que (el token de) "Scott $=$ Scott", de modo que el predicado "Jorge IV dudaba de que" se aplica respectivamente a 'decires" distintos en (1) y (3). En la medida en que consideramos un 'decir' (y una proposición) como una unidad no analizada, existe aquí un paralelismo superficial con Frege. 
Pero supongamos que alguien plantea la.cuestión siguiente: "En virtud del principio lógico de indiscernibilidad de los idénticos, se cumple que $\left[(x=y) . \emptyset_{x}\right] \supset \emptyset_{y}$; por lo tanto, el esquema de inferencia: " $x=y$; $\emptyset_{x} ; / \therefore \emptyset_{y}$ " es un esquema válido. Y el razonamiento: (1); (2) / $\therefore$ (3) parece tener la forma lógica autorizada por tal esquema; ¿cómo es entonces posible que las premisas sean verdaderas y la conclusión falsa?" Es ésta una pregunta muy de moda, y que ciertamente requiere una respuesta; ¿qué podría responderse desde el punto de vista de la teoría de los operadores?

Una posibilidad es decir que los verdaderos sujetos lógicos de (1) y (3) son las cláusulas subordinadas, de modo que la forma lógica de (1); (2); (3); / $\therefore$ (3) es realmente la exhibida por el esquema inválido:

(4). $\frac{x=y}{\emptyset p}$

donde " $x$ " $y$ " $y$ " son, como siempre, variables individuales, " $\emptyset$ " representa un predicado aplicable a 'decires', como "Jorge IV no sabía que", y " $p$ " y “ $q$ " representan 'decires' diferentes. Esto implica que las cláusulas subordinadas no son ahora analizables; pero ¿por qué? Después de todo, la aplicación de "Jorge IV no sabía que Scott $=\ldots$. a un nombre propio produce una oración verdadera-o-falsa y en esta medida se comporta como un predicado genuino.

El profesor Cohen dice que, en oposición a Frege, la teoría de los operadores mantiene la "interpretación normal" de los enunciados intensionales, y ello implica que no hay cambio alguno en la referencia de los nombres componentes, como ocurre en la teoría de Frege. Pero el hecho mismo de que "Scott" y "el autor de Waverley" conserven en ( 1 ) y (3) su referencia normal, en conjunción con la mencionada circunstancia de que la aplicación de "Jorge IV dudaba de que Scott $=$..." a "Scott" o "el autor de Waverley" produce una oración verdadera-o-falsa, hace aun más arbitraria la prohibición de considerar "Scott" o "el autor de Waverley" como. posibles sujetos lógi$\cos$ de (I) y (3); interesa observar de paso que esto no ocurre, por ejemplo, con la explicación de Quine, según la cual las figuraciones de "Scott" y "el autor de Waverley" en (1) y (3) son simplemente no referenciales.

En este punto Frege ofrece su teoría de la referencia indirecta, que nos permite mostrar que, sea cual fuere el modo en que analicemos (1) y (3), tomando en cuenta la estructura interna de las cláusulas subordinadas, la forma lógica de (1); (2) / $\therefore$ (3) no se ajusta al esquema del principio de indiscernibilidad. Esta teoría intenta: (i) explicar la conducta semántica de los nombres que componen la cláusula subordinada; (ii) dar el rango del predicado intensional "Jorge IV dudaba de que Scott $=\ldots$ ", evitando así 
que se lo rechace mediante un fiat arbitrario; y sea cual fuere el éxito final de la empresa, es ésta una ventaja incuestionable de la teoría de Frege. En relación con esto, Cohen dice simplemente que la ley de extensionalidad no vale ni siquiera en su forma más débil (cf. la introducción y $\S 1$ ), lo cual era de esperar, pues la forma débil de la ley de extensionalidad requiere algo análogo al cambio de referencia que encontramos en Frege. Pero tal situación requiere ser explicada, lo que Cohen no hace en parte alguna.

Antes de proseguir fundamentaremos brevemente, quizás de un modo demasiado sucinto, la anterior afirmación en favor de Frege. ${ }^{11}$ Como es bien sabido, desde el punto de vista fregeano la no extensionalidad es un caso especial de ambigüedad referencial. Lo que ocurre en (1) es que la cláusula subordinada, y por eso también los nombres componentes (incluyendo "=") denotan allí sus sentidos ordinarios (sentidos directos) y no sus denotaciones usuales. La cláusula subordinada como un todo denota una proposición (no, como usualmente, un valor veritativo) y los nombres componentes denotan los sentidos en cuya combinación consiste la proposición. Si suponemos ahora que el sentido directo de "Scott" es diferente del de "El autor de Waverley", se sigue que la sustitución que nos lleva de (1) a (3) cambia la denotación de la cláusula subordinada. Hasta este punto nos hemos ido aún más lejos que Cohen. Adoptaremos ahora algunas convenciones notacionales. Usaremos un nombre entre corchetes con un suscripto ${ }^{12}$ para denotar su sentido directo, por ejemplo "[Scott $]_{1}$ " para el sentido directo de "Scott", y la descripción estructural "([Scott $]_{1}[=]_{1}$ [el autor de Waverley $\left.]_{1}\right)$ " para representar la proposición denotada por la cláusula sųbordinada de (1). Entonces la forma de (1) podría hacerse más perspicua si escribimos:

(5) Jorge IV dudaba ([Scott $]_{1}[=]_{1}$ [el autor de Waverley $\left.]_{1}\right)$,

donde el prefijo oblicuante "que" ha desaparecido. A partir de (5) podemos obtener el predicado monádico:

(6) Jorge IV dudaba ([Scott $\left.]_{1}[=]_{1}[]_{1}\right)$,

donde "[ $]_{1}$ " indica un lugar a ser llenado por el nombre de un sentido individual. (6) es, en efecto, un predicado aplicable a sentidos (o conceptos, en la terminología de Church) individuales, y representa inequívocamente la propiedad expresada por el predicado natural "Jorge IV dudaba de que Scott $=\ldots$, que tiene la apariencia engañosa de ser un predicado de individuos.

11 Para más detalles, cf., mi artículo "Sobre la eliminación de los contextos oblicuos" (Critica, vol. 1, No 2, México, mayo de 1967).

12 La necesidad de un subíndice numérico surge del hecho de que en la teoría de Frege cada nombre posee un infinito número de denotaciones indirectas, correspondientes a la reiteración, potencialmente infinita, de operadores no extensionales. 
De manera que (5) nos permite resolver directamente el problema planteado por el principio de indiscernibilidad de los idénticos, mostrando que debido a la oblicuidad (= referencia indirecta) hemos confundido una propiedad de conceptos con una propiedad de individuos. Con esta interpretación puede decirse que la forma lógica de (1); (2); / $\therefore$ (3) es la exhibida por el esquema inválido:

(7) $\begin{aligned} & x=y \\ & \frac{\emptyset[x]_{1}}{\emptyset[y]_{1}}\end{aligned}$

y no la autorizada por el principio de indiscernibilidad. El hecho fundamental, en el cual consiste toda la diferencia entre (4) y (7), es que con esta interpretación no se requiere poner a la cláusula subordinada fuera del alcance del análisis lógico, como si fuese semánticamente indivisible; por el contrario, la teoría fregeana trata de revelar la conducta semántica de los términos singulares y predicados dentro de la cláusula subordinada de (1). No es mi intención afirmar que el aparato teórico de Frege es altamente creíble desde el punto de vista intuitivo; pero como Wilfred Sellars ha dicho ingeniosamente, al referirse a un análisis de tipo fregeano propuesto por él mismo: "The mechanics, if not the metaphysics, of the move, is comparatively straigthforward". ${ }^{13}$

Cohen tiene aún, sin embargo, la posibilidad de recurrir a una segunda estrategia. De acuerdo con su método de formalización (o Análisis 2; cf. la introducción) la verdadera forma lógica de (1) y (2) es tal que sus cláusulas subordinadas sólo aparecen como partes de un predicado aplicable a tokens; asi, (1) se transforma en:

(8) Hay un $x$ tal que $x$ es un 'decir' que Jorge IV dudaba de que Scott $=$ el autor de Waverley, etc.,

donde la frase subrayada expresa una propiedad atribuida al token $x$. No habría aquí, pues, ningún sitio apropiado para la sustitución de "el autor de Waverley" por "Scott". Pero es obvio que esta estrategia tampoco resuelve la cuestión fundamental: ¿son estos predicados no analizables, y por qué? Es sabido que las oraciones son analizables de diferentes modos: " $7>5$ ", por ejemplo, puede analizarse como " $(7)>(5)$ " o como " $(7>) 5$ " o como " $(>5)$ 7"; ¿por qué no es posible hacer lo mismo en el caso de oraciones que contienen estos peculiares predicados acerca de tokens? Cohen puede, naturalmente, decretar que en su lenguaje formalizado tales predicados son unidades analizables, y estaría en su derecho; pero difícilmente podría con-

13 "Some Problems about Belief" (Synthese, vol. 19, No 1/2, diciembre 1968, p. 164). 
siderarse esta decisión como una clarificación de un problema originado en el lenguaje ordinario.

(Nuevamente [cf. sección 2, más arriba] este punto es también relevante para el análisis nominalista de Scheffler; sus predicados formados por cláusulas que comienzan con "que" ['that' - clause predicates], como "que-Scott-esidéntico-al-autor-de-Waverley", deben considerarse como no analizables a fin de impedir que reaparezca la no extensionalidad. Pero Scheffler no pretende ocuparse de las fallas de la sustituibilidad; su propuesta está destinada a superar la especial objeción de Church a cualquier análisis nominalista de los enunciados de aseveración y creencia.) ${ }^{14}$

\section{Algo sobre modalidades}

Este rasgo insatisfactorio de la semántica de Cohen se hace aún más visible en su tratamiento de las modalidades lógicas, donde la promesa de una extensionalidad "mayor que la de Frege" se logra mediante la exclusión de predicados que contienen operadores modales, tales como "es necesariamente la estrella vespertina". Después de explicar al lector las conocidas dificultades asociadas con la cuantificación en contextos modales, Cohen nos dice:

Se sigue que en una formalización completamente extensional de la lógica general nunca podremos permitir que una expresión no extensional como "es necesariamente la estrella matutina" ocupe el lugar de una letra de predicado (p. 233).

Con este fin el autor introduce una estipulación (p. 213) que tiene el efecto de excluir los predicados de este tipo en la interpretación de su sistema formal. Refiriéndose al ejemplo

(48) La estrella matutina es necesariamente la estrella matutina, ${ }^{15}$

explica correctamente que la mencionada estipulación es "una consecuencia natural" de la teoría de los operadores, o sea

la teoría general de que todos los casos de no extensionalidad surgen [...] debido a la presencia de operadores que forman enunciados a partir de 'decires', y que por lo tanto pueden considerarse como generados por cierto tipo de enunciados acerca de 'decires'. Puesto que "necesariamente", aun cuando se halle insertada en el medio, opera sobre la totalidad del 'decir' referido, o sea sobre la totalidad de "La estrella matutina es la estrella matutina", debemos tratar (48) como un enunciado acerca de este 'decir', a saber, el enunciado de que es necesario, y

14 A. Church, “On Carnap's Analysis of Statements of Assertion and Belief" (Analysis, $\mathrm{XI}, 195^{\circ}$ ).

15 He conservado aquí la numeración de Cohen. 
no como un enunciado acerca de la estrella matutina, a los efectos de que tiene la propiedad de ser necesariamente la estrella matutina (pp. 233-34).

No objetaríamos demasiado este franco rechazo de las modalidades de re si Cohen no sostuviera al mismo tiempo que su teoría preserva "la interpretación normal" de estos enunciados (en otros términos: la referencia usual). Los argumentos esgrimidos en la sección precedente poseen también vigencia aquí, pero podemos completar nuestra crítica con la siguiente cuestión: si (48) es acerca del mencionado 'decir' (o sea "La estrella matutina = la estrella matutina"), debe hacer referencia a él de alguna manera; pero ¿cuál es aquí el mecanismo referencial? En la semántica de Frege la situación es clara: dentro de un contexto no extensional la cláusula subordinada es una combinación de nombres que denota, por correspondencia isomórfica, una combinación de sentidos, del modo explicado por la teoría de la referencia indirecta. Pero el profesor Cohen no gusta de este tipo de fantasias semánticas, y desea mantener la referencia normal de los nombres componentes; ¿cómo se explica, entonces, que (48) pueda hacer referencia al 'decir' como un todo y no a las cosas mencionadas por los nombres que lo integran?

\section{Extensionalidad y comillas}

En el $§ 25$ Cohen considera los enunciados:

y

(15) Giorgione era llamado así por ser gordo

(16) "Cicerón" contiene siete letras, ${ }^{16}$

que Quine presenta como típicamente no extensionales. El problema enfrentado por Cohen es la imposibilidad de conferir a (15) y (16) la forma general requerida por la teoría de los operadores, debido a que "ninguna cláusula subordinada ['that ...' clause] de tipo adecuado puede obtenerse de ellos en forma plausible" (p. 204). Coheñ siente que ésta es una dificultad crucial y el lector es puẹsto frente al dilema:

O bien estos enunciados nó son no extensionales en forma algụna, o deberemos abandonar [...] la' teoría de los operadores (p. 204).

Por supuesto, la respuesta casi inmediata es que "no son no extensionales", como el lector puede adivinar. Al negar el carácter no extensional de (16) Cohen argumenta que

16 Se sigue igualmente la numeración de Cohen, pero he modificado ligeramente el ejemplo para adaptarlo al castellano. 
en (16) no figura el término "Cicerón", sino sólo la palabra-del-lenguaje [the language-word] "Cicerón". De modo que si deseamos intercambiar en (16) nombres que posean idéntica referencia, no debemos poner "Tulio" en lugar de "Cicerón", remplazando así un enunciado verdadero por uno falso, sino, en cambio, "el apellido de Tulio" por "Cicerón"», remplazando así un enunciado verdadero por otro (p. 204).

Podemos suponer que al decir que el término "Cicerón" no figura en (16) el autor quiere decir que el token pertinente de "Gicerón" no cumple alli su función referencial normal, ni, en rigor, función referencial alguna; pero ésta es precisamente la explicación de la no extensionalidad de (16) propuesta por Quine. Curiosamente, en este punto Cohen recurre al apoyo de Frege:

Para decir lo mismo en terminología fregeana: en (16) no se hace referencia directa ni indirecta a Cicerón, sino sólo a la expresión "Cicerón" (p. 204).

Esta referencia a Frege es muy equívoca porque su teoría de la referencia indirecta fue concebida para salvar la ley de extensionalidad en un sentido importante de esta infortunada palabra. No me detendría en esta cuestión si no fuera por el hecho de que se halla conectada con una confusión común entre a) negar la no extensionalidad de un contexto y b) explicarla, y además, entre dos sentidos de "no extensionalidad".

¿En virtud de qué son clasificados ciertos contextos usualmente como no extensionales? Simplemente, en virtud de lo que prima facie se presenta como una falla de la sustituibilidad de expresiones co-designativas (y de la regla de generalización existencial). ${ }^{17}$ Llamaré a ésta "no extensionalidad 1", o sea la no extensionalidad representada por la violación del principio

(A) Si $\Gamma a=b 7$ es una identidad verdadera, entonces $a$ y $b$ son intercambiables en todos los contextos salva veritate,

cuando (A) es interpretada simpliciter, sin imponerle ninguna condición restrictiva. Este principio puede considerarse como expresando la correspondiente noción de extensionalidad 1. Pero existe otro principio, que expresa la versión débil o contextual de extensionalidad (cf. introducción) y que puede distinguirse como extensionalidad 2:

Si un nombre que ocupa una posición $x$ en una oración $S$ es rem(B) plazado por otro que posee en $x$ la misma denotación que posee el primero en esa posición, entonces el valor veritativo de $S$ permanece invariable.

17 Paso por alto aquí otra fuente de la noción de extensionalidad (y de no extensio- 
Desde un punto de vista fregeano, un enunciado puede ser no extensional 1, pero sí extensional 2 (iy todo enunciado es necesariamente extensional 2 según esta teoria!); sin embargo, la teoría de los operadores nos dice que no sólo (A), sino también (B), es un principio falso en los lenguajes naturales, pues se supone que los nombres preservan sus referencias normales en los contextos modales y psicológicos.

Ahora bien, es claro que en el nivel pre-analitico el problema por resolver es planteado por la falla de (A); es la falla de (A) lo que nos permite hablar de no extensionalidad de un modo teóricamente neutral; y un buen paso hacia la clarificación del problema consiste precisamente en hacer una distinción entre (A), que es falso, y (B), que no puede serlo. Sostener la falsedad de (B) es proponer una teoria, y una demasiado peligrosa para ser adoptada como criterio clasificatorio en el punto de partida; pues negar (B) es rechazar el principio de indiscernibilidad de los idénticos como fue visto en la sección precedente.

Yo sugeriría que la diferencia entre (16) y los contextos no extensionales (en el sentido de (A) ) $)^{18}$ como "X cree que..." es simplemente que (16) es un caso de no extensionalidad trivial, fácil de explicar mediante la distinción entre uso y mención, carente de implicaciones ontológicas perturbadoras y tal que es fácil construir una oración extensional equivalente; esta última puede obtenerse, por ejemplo, mediante el uso de nombres de letras y la operación de concatenación, como fue indicado por Quine (Word and Object, p. 143 ).

Es verdad que Quine dice que, debido a que disponemos de una formulación extensional,

cualquier figuración no referencial causada por la cita directa mediante comillas [by quotation] constituye un fenómeno de superficie [surface appearance], fácilmente eliminable mediante un cambio de notación (p. 143).

Y agrega en la página 212:

Siendo eminentemente opaca, la construcción mediante comillas es una forma vívida a la cual se pueden reducir otras construcciones opacas. Y es incluso posible eliminarla [dissolve] del todo, mediante el deletreo [spelling].

Pero ¿qué es "eliminar" una construcción de comillas? Supongamos que

nalidad), según la cual un enunciado es no extensional si hace referencia a entidades no extensionales. Podría ser éste el caso de "El concepto El autor de Waverley determina el concepto individual Scott", donde ninguna sustitución de "Scott" por una expresión co-designativa puede alterar el valor veritativo del enunciado.

18 En lo que sigue sólo me referiré a la no extensionalidad 1. 
en vez de «"Cicerón" escribimos, siguiendo la receta de Quine: "ceiceeereoene" y de este modo transformamos (16) en:

(17) ceiceereoene tiene siete letras.

En la página 143 Quine nos dice que el deletreo (combinado con la concatenación de los nombres de las letras) "es un procedimiento alternativo para la misma función que cumple la construcción mediante comillas". De modo que tenemos primero una construcción de comillas, que es opaca; y también una construcción diferente, que sirve los mismos propósitos que la anterior, pero no es opaca. Es obvio para mí que ambos tipos de construcción existen; no es necesario expulsar a una de ellas del reino de la realidad.

De hecho, la reducción extensionalista más usual de los enunciados no extensionales (por ejemplo, la efectuada por Carnap en Logical Syntax of Language) consiste en reformularlos por medio de comillas, o sea en reducir una no extensionalidad sospechosa a otra inocente.

A veces esta cuestión se complica aún más por el hecho de que algunos autores sostienen que ni siquiera la palabra "Cicerón" (en la terminología de Cohen: la palabra-del-lenguaje "Cicerón", o un token de ella) figura en (16), sino sólo la palabra «"Cicerón"»; al criticar a Quine, J. G. Kemeny dice, en efecto, que debido a ello la sustitución de "Cicerón" por "Tulio" en (16) "es imposible".19 La posición de Kemeny equivale a interpretar la figuración de "Cicerón" en (16) como un accidente ortográfico (análoga a la de "Juan" en "Juancho"); curiosamente, esta interpretación es también defendida por Quine mismo. Pero pasando por alto el hecho de que ésta no es la única, ni la mejor, interpretación de los contextos de comillas, la generalización de esta estrategia haría del todo imposible hablar de la falla de la sustituibilidad: podríamos decir, por ejemplo, que la palabra "Scott" que figura en "Scott $=$ el autor de Waverley" no es la misma que figura en " $\mathrm{X}$ cree que ... Scott...", siendo éste un caso especial de homonimia. ¿Guáles son, entonces, los contextos no extensionales?

Thomas M. Simpson

Consejo Nacional de Investigagiones Científicas y Técnicas Buenos Aires 